\title{
PATRIMONI CULTURALI E PIANIFICAZIONE DEL TURISMO IN ITALIA \\ E IN EUROPA
}

\section{CULTURAL HERITAGE AND TOURISM PLANNING IN ITALY AND EUROPE.}

Marina Faccioli

faccioli@uniroma2.it

Università di Roma Tor Vergata

\section{Sintesi}

In Europa la attuale pianificazione del turismo si ispira ai criteri che hanno guidato la programmazione territoriale. Gli elementi di base sono la coesione sociale fra i soggetti, la valorizzazione delle risorse radicate nelle tradizioni e nei patrimoni sociali e territoriali, la lotta contro lesclusione Non solo i monumenti e $\mathrm{i}$ beni culturali più conosciuti nel mondo sono i patrimoni su cui si vuole basare lo sviluppo del turismo. Il patrimonio di un territorio è soprattutto qualcosa di vivo, che deve essere protetto e ricostituito continuamente, perché viene riconosciuto nella storia della vita e del lavoro dei suoi abitanti.

Parole chiave: Patrimonio; Cultura; $\quad$ Territorio; Turismo; Città

\begin{abstract}
European current tourism planning is based on the criteria inspiring urban development planning. Its basic features are social cohesion, optimazation of tradition, as well as social and cultural heritage, rooted resources. Tourism development is not only to be based on the well-known artistic and cultural heritage, but, overridingly, on the land heritage, to be considered as a living being to be continuously protected and regenerated, as it mirrors its inhabitants? lives and work stories.
\end{abstract}

Key Words: Heritage; Culture; Territory; Tourism; Cities/Towns 
Nel corso dell'evoluzione del pensiero geografico avvenuta negli ultimi decenni, una sorta di "rivoluzione culturale" ha investito l'analisi delle relazioni fra geografia del turismo come conoscenza dei luoghi e dei processi turistici, da un lato, e studio del patrimonio territoriale, dall'altro. Operatori pubblici e privati, residenti, operatori economici, studiosi del territorio, pianificatori e fruitori delle risorse dei patrimoni territoriali, come turisti e ogni categoria di visitatori, hanno capito, per propria esperienza, come possa funzionare come luogo turistico solo un territorio che "vive di per sé", cioè non per le immagini, sempre più spesso falsificate, e gli "errori" che vengono dalle rappresentazione di certa politica turistica speculativa, ma piuttosto per il proprio spessore, per la coerenza con la propria storia. Solo in questo modo, è apparso evidente, i luoghi possono diventare, a ogni effetto, patrimoni territoriali costruiti e consolidati (Minca, 1996).

Patrimonio territoriale e sviluppo locale. - E' su queste basi che, credo, vada affrontato il tema del patrimonio culturale in Italia e in alcuni Paesi europei, secondo una rilettura di alcuni tipici processi che in quei Paesi hanno interessato ogni aspetto della vita sociale.

In Italia e in tutta l'Europa negli anni più recenti un "altro", originale patrimonio culturale si è andato costituendo, sulla base dei processi di sviluppo economico, sociale e culturale locale che si sono verificati, per lo più spontaneamente, a partire dagli anni Settanta e Ottanta del secolo passato. E' il patrimonio che si è progressivamente costruito nel quadro complesso della storia territoriale di questi Paesi.

Si è trattato di processi di forte crescita che hanno interessato piccole, spesso piccolissime, e medie imprese di carattere familiare, creando nuove tipologie di occupazione e di produzione dopo la crisi della grande impresa industriale seguita, in primo luogo, alle conseguenze dello shock petrolifero dei primi anni Settanta. Quelle imprese si impegnarono in attività di trasformazione radicalmente diverse da quelle che avevano interessato la grande industria di base. Le nuove attività produttive erano connesse a tradizioni locali già vive, in settori dell'artigianato e della manifattura dei beni di largo consumo, come diversi comparti della meccanica minore, il tessile, la lavorazione della pelle, la trasformazione e conservazione di prodotti dell' agricoltura.

In Italia, in particolare, questi processi hanno avuto una grande importanza perché hanno posto le basi di gran parte della attuale struttura produttiva. Le regioni italiane in cui questi processi sono avvenuti sono state inizialmente quelle del Centro-Nord e del Nord-Est, dove c'erano forti tradizioni in settori artigianali, agricoli o di piccola manifattura.

Fattore di importanza essenziale è stato l'avvento di un modo nuovo di produrre ricchezza e di una nuova cultura del lavoro e della produzione industriale. L'impresa è uscita dalla grande 
fabbrica e si è stabilita nella famiglia, con il lavoro dei giovani, degli anziani, delle donne, spesso di ragazzi ancora in età scolastica. Ed è stata, anche questa, una sorta di "rivoluzione sociale", quando i protagonisti, operai e lavoratori espulsi dal lavoro a causa della crisi delle grandi imprese, hanno messo insieme i propri risparmi e le proprie competenze e reinvestito in piccolissime attività proprie o nel lavoro per conto di altri, anch'essi, piccoli produttori. Perché si è iniziato a produrre su basi minime di investimento, anche solo utilizzando il lavoro della propria piccola famiglia e partecipando a un sistema di attività di produzione condiviso fra tante unità coordinate, secondo una estrema divisione delle mansioni e dei ruoli. Attraverso queste attività si sono sviluppate, da parte delle piccole imprese e dei lavoratori, relazioni che hanno prodotto le condizioni per un apprendimento progressivo di conoscenze, informazioni e competenze, insieme a un crescente radicamento nel proprio territorio, che ciascuno ha consolidato sul sistema dei valori della propria vita quotidiana.

Il più importante fra i modelli organizzativi che si sono affermati in quella fase è quello del "distretto industriale", un contesto territoriale formato da aree comunali contigue, animato da un processo di interazione fra produttori fondato sulla fiducia reciproca, sull'accettazione di regole comuni di convivenza nel territorio, in cui i soggetti assumevano una responsabilità e una identità collettiva, definendo condizioni stabili di concertazione fra il settore pubblico e quello privato.

Il processo di produzione come patrimonio. - Negli ultimi due decenni la produzione delle piccole e medie imprese si è trasformata profondamente orientandosi verso l'impiego delle tecnologie, dell'informazione, del capitale umano.

Tuttavia, dalla intera storia dello sviluppo locale, dalle performances di successo e, ancor più, dalle storie dei fallimenti e della crisi radicale del mondo distrettuale, fatti che hanno portato a un cambiamento di senso del concetto di locale e localismo, sono rimasti insegnamenti importanti nella cultura del nostro Paese. Insegnamenti e "moniti" derivati dalla verifica degli aspetti negativi della storia dei distretti, come il consumo spesso dispersivo di territorio, l'uso del credito mediante strumenti fuori mercato, la rincorsa al profitto centrata su carriere personali o familiari.

Le eredità distrettuali che ancora contano nella gestione del patrimonio territoriale guardano, invece, alla necessità della riconversione e della riconquista continua di modalità di lavoro e di produzione fondate sulla valorizzazione di "saper fare" radicati storicamente, sull'esigenza di una condivisione di regole che metta in primo piano il ruolo degli attori territoriali, su un apprendimento progressivo e collettivo, sulla massimizzazione della collaborazione e interazione a diverse scale, 
sulla consapevolezza, soprattutto, dell'esistenza di tante realtà "locali" quanti sono i percorsi di aggancio di queste a reti trans locali e internazionali.

A scala mondiale oggi la produzione industriale sta percorrendo più strade che, pur se per vie diversificate, portano a vicende di riconcentrazione su grandi percorsi di interesse sempre più articolato. Sono catene globali di produzione di valore, presentate da una vasta, recente letteratura soprattutto economica (Agostino e altri, 2010; Grossman and Rossi-Hansberg, 2006; Sturgeon, Van Biesebroeck and Gereffi, 2008)), all'interno delle quali va rapidamente crescendo il numero delle imprese che operano, pur in diversi settori, in posizione necessariamente intermedia, quasi secondo un allargamento progressivo e smisurato di comparti di subfornitura di scala mondiale. Diventano intermedie anche imprese distrettuali che fino a poco tempo fa si ponevano a diretto contatto con $\mathrm{i}$ mercati, come imprese finali, su cicli più brevi.

Oggi parlare di locale significa parlare di imprese e processi di produzione che sussistono solo se consapevoli di operare, in posizione intermedia, entro catene mondiali.

Nello stesso tempo, la produzione di scala locale rimane competitiva solo se originale, non standard, solo se è fondata su radicamento e identità territoriale, se è portatrice di un patrimonio territoriale consolidato e unico, dotato di valore e pregio manifatturiero/artigianale, storico, culturale o architettonico. E lavorare nel locale è diventato un modo specifico, e per più versi translocale, di riconoscere e partecipare ai processi che interessano il territorio e, insieme, una cultura della concertazione, di una nuova domanda di governance dei fatti territoriali che ha interessato gli assetti istituzionali e gli organismi delle amministrazioni locali. E significa, soprattutto, possedere una sensibilità che consenta, prima ancora che il poter produrre, il saper "catturare" nel modo vincente gli input intelligenti e trasferibili che vengono dalla storia vissuta dei territori, così da poterli analizzare, interpretare e comunicare a scale extralocali, per conoscere e gestire la cultura degli stessi territori e poter collaborare alla loro sopravvivenza.

Anche il patrimonio culturale è, progressivamente, divenuto "altro" rispetto alla convenzione per cui patrimonio erano prevalentemente i beni culturali, i grandi monumenti delle città d'arte italiane e europee. Oggi patrimonio significa anche molte cose diverse e tra loro interattive: produzioni tradizionali, ricorrenze e feste religiose e pagane, musica, enogastronomia, cultura ed educazione alimentare, artigianato, ma anche paesaggi, vita urbana e, più di tutto, esperienze di conoscenza profonda delle componenti essenziali e strategiche dei milieux, e dunque vita dei territori, ricordi, suggestioni e immagini.

Patrimonio è qualcosa da non confondere genericamente con la semplice risorsa territoriale: è il risultato di un processo continuo di "patrimonializzazione" cioè di ricostituzione e riproduzione 
delle componenti originali e uniche di un territorio, quelle che consentono che quel territorio continui a vivere. E il patrimonio va interpellato come soggetto vitale e vivente; va riprodotto, perché non muoia, attraverso la creazione continua di "valore aggiunto territoriale" come garanzia vera di sostenibilità (Dematteis, 2001).

In questi termini, patrimonio è oggi ogni modo di sentire, di agire, produrre e governare che si sia consolidato nella storia di un territorio e che abbia acquistato una funzione specifica e insostituibile nella ricostituzione continua delle caratteristiche essenziali e inprescindibili del territorio medesimo.

Dunque è patrimonio anzitutto una dimensione di comportamento che diviene "politica", cioè la "territorialità" intesa come appartenenza al luogo geografico, vissuta in forma attiva, cioè collettiva, dunque rivolta alla produzione di bene comune, per molti aspetti eredità forte, pur rivista e trasformata, della storia dello sviluppo locale nel nostro Paese.

Il Piano Strategico per il Turismo della Provincia di Novara. - Ed è patrimonio l'intero bagaglio della vita di un territorio. Le città italiane del Nord-Ovest, le prime che hanno vissuto, consolidato e poi visto entrare in crisi uno sviluppo industriale ormai maturo, legato alla grande impresa, stanno vivendo, già da alcuni anni, una stagione di trasformazione della propria base produttiva, sociale e culturale e l'esigenza di trasformare e riproporre la propria immagine, per diventare attrattive, interessanti per il viaggiatore d'affari, che è stato finora il più abituale fra $i$ propri visitatori, ma anche per ogni altra tipologia di viaggiatore e turista.

Si tratta di riproporre la propria immagine in linea con i nuovi orientamenti dell'industria, dell'economia e della cultura complessiva dei territori, con le filiere globali della produzione e della socialità, e dunque in sintonia con le attuali strade percorse dalla politica di programmazione territoriale. Oggi, ancor più che produrre ricchezza, è importante cogliere, acquisire e saper valorizzare la ricchezza già accumulata nelle tradizioni più competitive, il valore intrinseco dei patrimoni ereditati, la qualità culturale depositata in tutto quello che si è costruito sapientemente nei nostri territori, per trasformarla in ricchezza sociale condivisa, in patrimonio collettivo, in bene comune fruito dai diversi soggetti che nel territorio operano, con diversi ruoli e a diverse scale.

Le nostre città vogliono "risignificarsi" per motivi di mercato ma anche e soprattutto per partecipare di una nuova cultura, diventando luoghi in cui sia possibile, e piacevole, fermarsi, in cui vivere, e magari mangiare, bene, in cui apprezzare il paesaggio, la qualità dell'ospitalità e dell'accoglienza, l'offerta storico-artistica, lo shopping e soprattutto la qualità della vita urbana. Luoghi in cui la cultura diventi anzitutto scambio, interculturalità, cosmopolitismo, connessione e 
interazione continua fra $\mathrm{i}$ popoli. $\mathrm{E}$ in queste città gran parte dei capitali già accumulati dall'industria vengono reinvestiti dagli imprenditori per promuovere Piani di valorizzazione e riqualificazione territoriale e, in particolare, Piani Strategici di Sviluppo del Turismo.

Il patrimonio su cui investire è in questi nuovi Piani il tessuto sociale, spaziale, produttivo della vita quotidiana di queste città. E' la base economica, politica, culturale nel senso più ampio, su cui le stesse città si sostentano e investono per il proprio futuro, fatta di progetti di ristrutturazione industriale, infrastrutturale e logistica, di internazionalizzazione delle relazioni di mercato, di sviluppo di servizi innovativi e specialistici, di rinnovamento delle volontà e capacità di comunicazione da parte della società locale: tutto questo, agito e promosso grazie all'intervento congiunto di operatori privati e pubblici, soggetti individuali e istituzioni, tour operators, residenti, visitatori impegnati a "fare sistema" nell' ambito di esperienze di governance condivisa.

Novara, una delle principali, vecchie città industriali del Piemonte Orientale, situata vicino al confine fra le regioni del Piemonte e della Lombardia, contigua all'area metropolitana di Milano, vanta un Piano d'Azione per lo sviluppo della promozione, dell'accoglienza e dell'informazione turistica che esamina, in questa chiave, le caratteristiche del movimento turistico provinciale e le relative prospettive di sviluppo individuando, a fianco dei tradizionali flussi di visitatori business, alcuni segmenti potenziali di riqualificazione o di sviluppo turistico in linea con le complessive prospettive di cambiamento territoriale locale, cioè:

il "turismo culturale o d'animazione collettiva", composto da turismo naturalistico, storicoreligioso, sportivo ed eno-gastronomico. E' un segmento che trae importanza dalla particolare struttura urbana della Provincia, inserita nel punto di passaggio fra la densità metropolitana milanese e il tipico sviluppo "periurbano" locale che offre servizi e vita urbana senza perdere i vantaggi della campagna, con terreni coltivati, agricoltura e orti. I fruitori di questo segmento di offerta turistica sono soprattutto giovani impegnati in lavoro qualificato, che possono godere di una crescente quantità di tempo libero e di un buon livello di informazione;

il "turismo d'affari", a vantaggio di figure che godono di una elevata capacità di spesa, perché i loro spostamenti sono finanziati da società e imprese professionali, e di una crescente accessibilità a fonti di comunicazione e informazione;

il "turismo indotto dall'intermodalità trasportistica" che sfrutta la posizione della città di Novara all'incrocio fra direttrici internazionali, un asse verticale che congiunge il Centro-Nord europeo con il bacino del Mediterraneo, e un asse orizzontale, da Ovest a Est, fra Barcellona e Kiev passando, in Italia, per Torino, Milano, Trieste;

Geo UERJ - Ano 14, no. 23, v. 2, $2^{\circ}$ semestre de 2012 p. 362-374 
il "turismo d'animazione della città universitaria" che attrae studenti da tutto il Piemonte, decongestionando l'università di Torino, e dall'area milanese. Questo segmento offre servizi, di ricettività e non solo, specifici per i giovani, che vanno a costituire un patrimonio molto interessante destinato a rinnovare e attualizzare la domanda turistica complessiva;

il "turismo internazionale" riferito alle opportunità che vengono al territorio novarese da eventi extralocali, come l'organizzazione delle Olimpiadi invernali di Torino del 2006. In quell'occasione la città di Novara era stata coinvolta marginalmente, trovandosi come punto di passaggio intermedio fra Torino e Milano. A causa dell'importanza mondiale dell'evento olimpico, tuttavia, gli effetti in termini di comunicazione sono rimasti a lungo, così da attrarre visitatori, soprattutto internazionali, intorno alle strutture programmate e utilizzate nei periodi successivi all'evento stesso per gli sport invernali. In questo caso l'evento olimpico ha consentito la creazione e la riqualificazione di nuovi originali patrimoni da utilizzare a fini turistici.

La particolarità del Piano è, complessivamente, nell'aver promosso immagini, brand territoriali che integrino le componenti dell'offerta, non più settori turistici singoli, legati a risorse stereotipate (mare, montagna, città d'arte). La Provincia novarese, infatti, punta sulla riqualificazione di risorse che in passato nulla avrebbero avuto a che spartire con il turismo (come già visto, ristrutturazione di apparati industriali, infrastrutture per la comunicazione, presenza dell'università, uso di paesaggi agricoli..) e che invece oggi possono attrarre investimenti, entrare in cicli di produzione di servizi innovativi e specialistici, mirati per partecipare a filiere turistiche di interesse mondiale.

In questo senso si pone il capoluogo, a cui la crescita dell'escursionismo legato al lavoro e agli affari assegna un ruolo sempre più competitivo in termini di capacità attrattiva. Novara, infatti, città d'affari, avverte sempre di più l'esigenza di proporsi come meta turistica, sfruttando le proprie risorse tipiche. Si pone dunque come una "periferia" milanese, non più subalterna a Milano ma fortemente riqualificata; come terminal aeroportuale, per la vicinanza all'aeroporto internazionale di Malpensa; e come sede di un turismo "di nicchia" che guarda a risorse ambientali poco note ma altamente fruibili. Il territorio che circonda la città è ricco di beni culturali "minori" ma estremamente interessanti, di paesaggi tipici della Pianura Padana, di vigneti coltivati nelle Colline Novaresi, dove si trovano qualità di vini di altissimo livello e prodotti enogastronomici di pregio.

Una risorsa particolarissima, un paesaggio tipico dell'Italia del Nord, su cui si sta puntando per trarne un brand turistico interessante, già apprezzato soprattutto da visitatori stranieri, è la storica risaia, l'area di coltivazione del prezioso riso piemontese, che nella stagione primaverile offre uno spettacolo unico, un fitto prato erboso che emerge dai bacini d'inondazione, una distesa 
verde che sembra sospesa sull'acqua. La conduzione della risaia ha creato una infrastruttura insediativa che, nel tempo, è diventata un soggetto territoriale a sé stante, interconnesso fortemente con più forme di urbanizzazione locale. Attualmente la produzione di riso italiano è in crisi e, allora, l'ecosistema della risaia, con la sua storia, il suo regime fondiario e i suoi paesaggi è, nel tempo, diventato "altro" e oggi si propone come risorsa attrattiva, come un contesto interessante e affascinante da visitare piuttosto che, come in passato, solo come sistema economico-produttivo.

Dunque, patrimonio essenziale da sfruttare per creare e incrementare processo turistico è, per la provincia di Novara, la conoscenza e l'apprezzamento del contesto ambientale nel suo significato più complesso.

Oggi si deve far conoscere e valorizzare la campagna della Pianura Padana, i beni culturali poco conosciuti, il vino e la cucina dei piccoli centri e della vecchie tradizioni, i territori, come la risaia, da conoscere passeggiando "lentamente", i paesaggi che si presentano negli scorci fra la pianura, la collina e lo sfondo delle Prealpi e delle Alpi, le risorse apparentemente "minori", che si possono godere nella vita quotidiana. Sono queste le risorse che devono diventare patrimonio locale su cui investire per creare turismo. Non conta più, come era in passato, solo la presenza del Lago Maggiore o dei beni culturali presenti nel capoluogo, cioè le risorse locali più conosciute ma ormai anche le più "invecchiate", che hanno prodotto notevole turismo internazionale e oggi sono in declino proprio perché non hanno finora rinnovato le proprie strutture e le proprie tipologie di offerta.

La programmazione turistica, il processo urbano e le istituzioni. - Le risorse essenziali del turismo non sono più, dunque, solo quelle "date" dalla natura o dalla storia, non bastano più le "rendite di posizione", né i soli prodotti dell'industria manifatturiera. Servono patrimoni radicati e consolidati, tuttavia riproposti attraverso le tecnologie, le competenze immateriali e conoscitive; patrimoni assolutamente originali e unici, che provengono dalla cultura, dall'ambiente, dalla creatività come risorsa strategica tipicamente italiana, dall'italian way of life.

Nella pratica della programmazione rivolta alla identificazione e qualificazione dei patrimoni territoriali originali e allo sviluppo del processo turistico, "fare sistema" significa portare avanti congiuntamente un'azione volta all'interesse collettivo, da parte di produttori che devono "saper" comunicare la tipologia dell'offerta territoriale locale, e consumatori/turisti che devono "saper" partecipare di suggestioni ed esperienze, costruire immagini e saper raccontare esperienze e immaginari costruiti durante quelle esperienze. Perché è la formazione rivolta a "saper fare turismo" da parte di operatori e fruitori, alla pari, l'obiettivo importante per chi vuole "saper vivere" nel 
turismo. Bisogna aver imparato a fare esperienza, bisogna conoscere le nuove pratiche, bisogna "essere capaci di partecipare", non basta più solo spendere denaro. E bisogna saper fare e ascoltare racconti delle esperienze vissute. Perché un territorio tanto più conta come prodotto turistico quanto più viene raccontato e perché è nell'esperienza che si riesce a fare sistema ed è dunque la stessa esperienza che diviene il vero patrimonio culturale della società che vive in un territorio.

Questi contenuti e queste rinnovate modalità di produzione e di comportamento si sono posti nel corso dell' ultimo decennio alla base della politica di intervento territoriale locale in Italia, come negli altri Paesi europei, in forme che sono state sancite dalle istituzioni, nel Quadro Strategico Nazionale per quanto riguarda l'Italia e, a scala europea, nello Schema di Sviluppo dello Spazio Europeo e in particolare, in un'ottica specificamente pertinente all'analisi geografica del patrimonio, nella Convenzione Europea sul Paesaggio che ha promosso il paesaggio come soggetto primario della politica territoriale e come eccellenza fra i patrimoni culturali territoriali a scala internazionale.

Il Quadro Strategico Nazionale (QSN), approvato dalla Commissione Europea nel 2007, riguarda obiettivi, priorità e regole relativi alla politica regionale di sviluppo prevista per l'Italia nell'arco di tempo compreso fra il 2007 e il 2013. Il documento, specificamente orientato ad accrescere la qualità, la sicurezza e l'inclusione sociale nei territori, insieme garantendo competitività e attrattività delle città e dei sistemi urbani, dedica una specifica priorità al turismo (1)

Gli obiettivi specifici riguardano:

la valorizzazione della rete ecologica e la tutela della biodiversità per promuovere condizioni di sviluppo economico sostenibile;

la valorizzazione dei beni e delle attività culturali come vantaggio comparato delle regioni italiane per migliorare la qualità della vita di residenti e visitatori, accrescendo l'attrattività dei luoghi e potenziando, negli stessi luoghi, la coesione sociale;

l'incremento della competitività internazionale delle destinazioni turistiche delle regioni italiane, attraverso un miglioramento del posizionamento dei prodotti turistici territoriali nel mercato;

il rafforzamento delle capacità di conservazione e gestione dei patrimoni naturali/paesaggistici e culturali attraverso azioni di cooperazione territoriale.

Anche progetti istituzionali di interesse internazionale, come lo Schema di Sviluppo dello Spazio Europeo, propongono alcuni principi di politica turistica che guardano essenzialmente alla qualificazione e rivitalizzazione del patrimonio come dato socio-territoriale. Fra questi principi, la coesione territoriale, il riconoscimento del ruolo delle città, il miglioramento dell'accessibilità 
urbana (fisica, infrastrutturale e economico-culturale), lo sviluppo endogeno, una moderna partnership fra città e campagna, la tutela/gestione dei patrimoni paesistico-culturali.

Nel 2005, nel quadro di una revisione del testo del Trattato di Lisbona, l'Unione Europea ha riconosciuto un ruolo primario e strategico al turismo come fonte di ricchezza economica e culturale per una crescita sostenibile che conferisca maggiore qualità al lavoro e come incentivo alla convivenza e coesione fra i popoli e, dunque, alla costruzione europea.

Lo stesso Schema di Sviluppo dello Spazio Europeo promuove come destinazioni europee di eccellenza, in chiave di cooperazione internazionale, alcuni insiemi territoriali quali modelli di benchmarking, sulla base di una piattaforma collaborativa interistituzionale e transfrontaliera:

l'Arco Latino, come partnership nel Mediterraneo occidentale, formato da partners Italiani (province di Alessandria, Asti e Torino), Francesi e Spagnoli;

l'Euroregione Alpi Mediterraneo, ancora un'euroregione a base mediterranea, formata dalle regioni italiane Piemonte, Liguria, Val d'Aosta e le regioni francesi Rhone-Alpes e ProvenceAlpes-Cote d'Azur.

L'Arco Latino, istituito alla fine degli anni Novanta grazie al patrocinio della politica regionale europea e del Conseil Général des Alpes Maritimes, è rivolto a creare una partnership nel Mediterraneo occidentale che funzioni come rete strategica per lo sviluppo locale, in particolare turistico, sulla base di partenariati territoriali di carattere trasfrontaliero, interregionale e transnazionale. Comprende un'area con oltre 70 milioni di residenti in cui i membri attivi, costituiti soprattutto da attori socio-economici e imprese piccole e medie, lavorano per favorire una stabile cooperazione e integrazione fra l'Arco Latino e l'intera area del Mediterraneo.

L'Euroregione Alpi Mediterraneo, attiva dal 2006, abitata da oltre 17 milioni di residenti, è strettamente inserita, per la sua posizione e la sua storia, nella complessa geografia del massiccio alpino e delle relazioni che questo intrattiene con il Mar Mediterraneo. Tra le finalità specificamente rivolte al turismo vi sono la realizzazione di politiche per la destagionalizzazione dei flussi di visitatori mediante la promozione di turismo di prossimità fra i territori dell'Euroregione; lo scambio di best practices nell'ecoturismo, nel turismo accessibile e per il sostegno dell'occupazione e la messa a punto di nuovi strumenti informativi; e la collaborazione, mediante lo scambio di metodologie di analisi e ricerca, fra osservatori turistici regionali.

In entrambi i casi un ruolo forte è sostenuto dalle città che in queste aree si trovano al confine tra Francia, Italia e Spagna, quasi a promuovere una funzione specifica delle stesse montagne di confine. Città e montagne si propongono, infatti, nello stesso tempo, come aree turistiche altamente competitive e come nodi economici, infrastrutturali e interculturali di 
importanza internazionale, così da conferire alle stesse linee di frontiera fra i Paesi un ruolo che favorisce l' incontro e la relazione fra le diverse società, piuttosto che una funzione di separazione.

In questo senso la città, o piuttosto i multiformi processi di urbanizzazione che la tradizione geografica percepisce come strategici campi relazionali in cui il locale e il globale si interfacciano, in più casi anche conflittualmente, si ripropongono come soggetti territoriali "eccellenti", campo di incontro delle più idonee e aggiornate politiche di gestione del patrimonio territoriale e culturale e soggetti di interesse primario per ogni politica di sviluppo del turismo.

\section{NOTE}

(1) La priorità 5 è dedicata al turismo e individua gli indirizzi rivolti a "trasformare in vantaggio competitivo l'insieme delle risorse naturali, culturali e paesaggistiche locali per aumentare l'attrattività anche turistica del territorio, migliorare la qualità della vita dei residenti e promuovere nuove forme di sviluppo economico sostenibile" (Commissione Europea, 2007).

\section{BIBLIOGRAFIA}

M. Agostino e altri, L’impresa manifatturiera subfornitrice italiana nella catena del valore globale. Un confronto di produttività, in A. Zazzaro (2010), pp. 199-227.

Centro Studi Confindustria, Effetti della crisi, materie prime3 e rilancio manifatturiero. Le strategie di sviluppo delle imprese italiane, "Scenari industriali", 2011, 2.

F.M. Coccia e G. A. Cocco, Impresa Turismo, Roma, Unione Italiana Camere di Commercio, Industria, Artigianato e Agricoltura, 2012.

Commissione Europea, Comunicazione della Commissione al Parlamento Europeo, al Consiglio, al Comitato Economico e Sociale Europeo e al Comitato delle Regioni. Agenda per un turismo europeo sostenibile e competitivo, Bruxelles, 2007. 
S. Conti, E. Dansero e F. Sforzi, Environment, Innovation and Reorganisation of Industrial Space. A theoretical framework, "Bollettino della Società Geografica Italiana", 1996, 1, pp. 45-64.

E. Dansero, C. Emanuel e F: Governa (a cura di), I patrimoni industriali. Una geografia per lo sviluppo locale, Milano, Angeli, 2003.

G. Dematteis, Per una geografia della territorialità attiva e dei valori territoriali, in P. Bonora (a cura di), SLoT Quaderno 1, Bologna, Baskerville, 2001, pp. 11-30.

C. Emanuel e R. Afferni, Piano d'Azione per lo sviluppo della promozione, dell'accoglienza e dell'informazione turistica nella Provincia di Novara, Vercelli, Edizioni Mercurio, 2009.

C. Emanuel, S. Cerutti e M.E. Rossi, Progettare il cambiamento. Filiere programmatiche $e$ iniziative di sviluppo turistico in Piemonte, Vercelli, Edizioni Mercurio, 2011.

M. Faccioli, Nuove centralità territoriali e "produzione" culturale: la rivalorizzazione di luoghi turistici nell'area romana, in P. Brandis e G. Scanu (a cura di), La Sardegna nel mondo mediterraneo: $V^{\circ}$ Convegno internazionale di studi, vol. 13, L'importanza economica del turismo oggi, Bologna, Pàtron, 2001, pp. 375-386.

M. Faccioli, Territorio, città d'arte e produzione di patrimoni culturali, in P. Morelli (a cura di), Beni culturali e turismo nelle città d'arte italiane, Milano, Angeli, 2003, pp. 25-40.

M. Faccioli, L'analisi territoriale dei processi turistici, in A. Pasqualini (a cura di), Il Turismo Culturale in Italia fra tradizione e innovazione, Roma, Memorie della Società Geografica Italiana, vol. LXXIX, 2005, pp. 251-270.

M. Faccioli, Territori locali e cultura globale, in C. Madau (a cura di), Risorse culturali e sviluppo locale, vol. I, Roma, Società Geografica Italiana, 2004, pp. 27-45.

M. Faccioli, Processi territoriali e nuove filiere urbane, Milano, Angeli, 2009. 
M. Faccioli, Filiere urbane o nuovi percorsi distrettuali? Cenni per una proposta di metodo, “documenti geografici”, Università degli Studi di Roma “Tor Vergata”, 2011, 16, pp. 5-21.

A. Giannola, Reti, distretti, filiere. Le problematiche fondamentali dello sviluppo italiano, in A. Zazzaro (2010), pp. 23-48.

G. Grossman and E. Rossi-Hansberg, The rise of offshoring: It's not wine for cloth anymore, Kansas City, Kansas Fed's Jackson Hole, 2006.

A. Lanzani, In cammino nel paesaggio. Questioni di urbanistica e geografia, Roma, Carocci, 2012.

C. Minca, Spazi effimeri. Geografia e turismo fra moderno e postmoderno, Padova, CEDAM, 1996.

C. Minca (a cura di), Lo spettacolo della città, Padova, CEDAM, 2005.

E. Rullani, Network Economy: Evolution of Small Firm Capitalism and of the "Made in Italy Sector”, "Economia e Politica Industriale", 2010, 2, pp. 141-165.

T. Sturgeon J. Van Biesebroeck and G. Gereffi, Value chains, networks and clusters: Reframing the global automotive industry, "Journal of Economic Geography”, 2008, 8, pp. 297-321.

C. Trigilia, Sviluppo locale. Un progetto per l'Italia, Roma-Bari, Laterza, 2005.

A. Zazzaro, Reti d'imprese e territorio. Tra vincoli e nuove opportunità dopo la crisi, Bologna, Il Mulino, 2010.

Artigo encaminhado para publicação em dezembro de 2012.

Artigo aceito para publicação em dezembro de 2012.

Geo UERJ - Ano 14, nº. 23, v. 2, $2^{\circ}$ semestre de 2012 p. 362-374

ISSN: 1415-7543 E-ISSN: 1981-9021

http://www.e-publicacoes.uerj.br/index.php/geouerj 